\title{
artigo
}

\section{Gravidez na adolescência e a assistência de enfermagem: uma abordagem sobre os ríscos à saúde maternal e neonatal}

Adolescent pregnancy and nursing care: an approach to the risks to maternal and newborn health Embarazo adolescente y cuidados de enfermería: una aproximación a los riesgos para la salud materna y neonatal

\begin{abstract}
RESUMO
Objetivo: Abordar os riscos existentes em gestações na adolescência que podem comprometer a saúde materna e neonatal, e o papel do enfermeiro na assistência de enfermagem, visando minimizar tais riscos. Método: A presente pesquisa trata-se de uma revisão sistemática da literatura, de característica exploratória, na qual buscou gerar dados quantitativos e qualitativos das amostras coletadas. Resultado: A análise dos estudos permitiu a sistematização e integração discursiva dos dados em três categorias: a) gravidez na adolescência e a assistência de enfermagem; b) gravidez na adolescência e os riscos à saúde maternal e neonatal; c) gravidez na adolescência e a prevenção dos riscos à saúde da mãe e do recém-nascido. Conclusão: Considerando a importância de reconhecer as questões mais relacionadas à saúde dos adolescentes, é possível concluir que o profissional de saúde, através da assistência de enfermagem, possui um papel fundamental de aconselhamento, educação e tomada de medidas preventivas.

DESCRITORES: Gravidez na adolescência. Educação em saúde. Riscos à saúde.
\end{abstract}

\section{ABSTRACT}

Objective: To address the risks that exist in teenage pregnancies that can compromise maternal and neonatal health, and the role of nurses in nursing care, aiming to minimize such risks. Method: The present research is a systematic review of the literature, with an exploratory characteristic, in which it sought to generate quantitative and qualitative data from the collected samples. Result: The analysis of the studies allowed for the systematization and discursive integration of data in three categories: a) teenage pregnancy and nursing care; b) teenage pregnancy and the risks to maternal and neonatal health; c) teenage pregnancy and the prevention of health risks for mothers and newborns. Conclusion: Considering the importance of recognizing the issues more related to the health of adolescents, it is possible to conclude that the health professional, through nursing assistance, has a fundamental role in counseling, education and taking preventive measures.

DESCRIPTORS: Pregnancy in adolescense. Health education. Health risk.

\section{RESUMEN}

Objetivo: Abordar los riesgos que existen en los embarazos de adolescentes que pueden comprometer la salud materna y neonatal y el rol del enfermero en el cuidado de enfermería, buscando minimizar dichos riesgos. Método: La presente investigación es una revisión sistemática de la literatura, con carácter exploratorio, en la que se buscó generar datos cuantitativos y cualitativos a partir de las muestras recolectadas. Resultado: El análisis de los estudios permitió la sistematización e integración discursiva de datos en tres categorías: a) embarazo adolescente y cuidados de enfermería; b) embarazo adolescente y riesgos para la salud materna y neonatal; c) embarazo adolescente y prevención de riesgos para la salud de madres y recién nacidos. Conclusión: Considerando la importancia de reconocer los temas más relacionados con la salud de los adolescentes, es posible concluir que el profesional de la salud, a través de la asistencia de enfermería, tiene un rol fundamental en la asesoría, educación y toma de medidas preventivas.

DESCRIPTORES: Embarazo en adolescencia. Educación em salud. Riesgo a la salud.

RECEBIDO EM: 31/10/2020 APROVADO EM: 19/11/2020 


\section{Mikael Henrique Jesus Batista}

Enfermeiro do Instituto Federal do Tocantins - Campus Colinas do Tocantins; Especialista em Urgência e Emergência pelo CGESP; Especialista em Terapia Intensiva Geral pelo CGESP; Mestre em Ensino em Ciências e Saúde pela Universidade Federal do Tocantins; Doutorando em Engenharia Biomédica pela Universidade Brasil; Professor do curso de enfermagem da Faculdade de Colinas do Tocantins - Grupo UNIESP.

ORCID: https://orcid.org/0000-0002-9277-8295

\section{Dabylla Bezerra Lino}

Acadêmica de enfermagem da Faculdade de Colinas do Tocantins - Grupo UNIESP. ORCID: 0000-0003-1170-8541

\section{Marluce de Sousa da Silva}

Acadêmica de enfermagem da Faculdade de Colinas do Tocantins - Grupo UNIESP. ORCID: 0000-0003-0272-6465

\section{Miquely Dayane Castro Costa}

Acadêmica de enfermagem da Faculdade de Colinas do Tocantins - Grupo UNIESP. ORCID: 0000-0003-2087-0161

\section{Marilene Alves Rocha}

Enfermeira do Município de Colinas do Tocantins, Tocantins; Mestra em Ciências Ambientais pela Universidade Brasil; Coordenadora do curso de enfermagem da Faculdade de Colinas do Tocantins - Grupo UNIESP.

ORCID: 0000-0003-4361-7011

\section{Tainá Soares Nunes}

Enfermeira do Município de Porto Nacional, Tocantins; Coordenadora da Unidade Mista Portal do Lago, distrito de Luzimangues, Porto Nacional.

ORCID: 0000-0002-0614-3841

\section{INTRODUÇÃO}

A adolescência é uma fase da vida humana, caracterizada por inúmeras alterações psicológicas, sociais, anatômicas e metabólicas, que deixam o ser humano em um estado desconhecido, é nesta fase que ele estipula referências para uma parte de sua vida adulta. Essas referências se definem na família, na escola e na sociedade, onde os adolescentes se inspiram para o desenvolvimento da sua personalidade ${ }^{1}$.

Nos últimos anos, o tema que aborda a gravidez na adolescência tornou-se uma importante discussão e alvo de políticas públicas em todo o mundo ${ }^{2}$. Considerando que três em cada dez adolescentes iniciam a vida sexual entre 13 e 15 anos $^{3}$, isto reflete na saúde dos mesmos, os quais podem contrair desde infecções sexualmente transmissíveis até uma gravidez precoce, não planejada, e de risco 4 .

Segundo a Sociedade Brasileira de Pediatria existe uma elevada taxa de gestação na adolescência no Brasil em comparação com a América Latina, sendo 400 mil casos/ ano, e quando relacionado à idade dessa população, o Ministério da Saúde refere que no ano de 2014 nasceram 28.244 filhos de meninas entre 10 e 14 anos e 534.364 crianças de mães com idades entre 15 e 19 anos. Já no ano subsequente, $18 \%$ dos brasileiros nascidos vivos eram filhos de mães adolescentes. Quanto à distribuição demográfica, a região com maior número de mães adolescentes é a região Nordeste, com 180 mil nascidos ou 32\% do total, seguido da região Sudeste, com 179,2 mil (32\%), a região Norte com 81,4 mil (14\%), a região Sul (62.475 - 11\%) e a Centro Oeste $(43.342-8 \%)^{26}$.

Tais dados são alarmantes e promovem preocupação, pois, a adolescência e a gravidez são estágios importantes para o progresso pessoal e da continuidade humana. No entanto, quando ambos estágios ocorrem ao mesmo tempo, pode desencadear uma desestruturação no desenvolvimento deste indivíduo, visto que, a gravidez pre- coce requer rigorosas cargas emocionais, físicas e sociais, pulando as etapas importantes de maturidade psicológica e sexual, constituindo comumente um problema de saúde pública no Brasil5.

Tratar a gravidez como uma condição de risco e de consequências adversas nesta fase da adolescência, é de suma importância e que pode reduzir agravos durante este processo, pois, as condições de proteção podem minimizar a vulnerabilidade $\mathrm{da}$ mãe e do recém-nascido ${ }^{6}$. Com base no declarado, a gravidez precoce, na adolescência, é tratada como um problema de saúde pública, e todas as ações tomadas devem ser analisadas de maneira ampliada, com o intuito de circundar a mãe adolescente e a criança recém-nascida, e os riscos que as cercam? ${ }^{7}$.

Em meio a um grande número de casos de gravidez na adolescência, muitos destes levam à complicações graves e até a morte de gestantes ou dos recém-nascidos $^{6}$. Diante disto, o presente estudo possui uma discussão atual e relevante, 
visto que ao fazer uma análise sobre os riscos que esse fenômeno pode oferecer para as adolescentes gestantes, possibilitará ressaltar o que já se compreende sobre o assunto, ou ainda, o que há de novo sobre a temática ${ }^{8}$.

Viso que, está fase da vida, compreendida pela adolescência com constantes mudanças e adaptações, a gravidez torna-se um risco para a saúde das mães e dos recém-nascidos, podendo ocasionar problemas como parto prematuro, anemia, aborto espontâneo, eclampsia e depressão pós-parto?. Deste modo, o presente trabalho traz como objetivo abordar os riscos existentes em gestações na adolescência que podem comprometer a saúde materna e neonatal, e o papel do enfermeiro na assistência de enfermagem, visando minimizar tais riscos.

\section{MÉTODOS}

A presente pesquisa trata-se de uma revisão sistemática da literatura, de característica exploratória, na qual buscou gerar dados quantitativos e qualitativos das amostras coletadas. Esta pesquisa utilizou de procedimentos técnicos, que possibilitasse reunir informações e dados que auxiliasse na fundamentação teórica, delimitação da problemática, que atendesse a hipótese estabelecida e ao objetivo proposto $^{10}$.

Com o propósito de atender ao objetivo da pesquisa, o método para execução deste trabalho foi constituído por algumas etapas distintas, seguindo o protocolo específico proposto na literatura, como: delimitação da questão a ser tratada na revisão; definição das palavras chaves; seleção das bases de dados bibliográficos para consulta e coleta do material; definição dos critérios de inclusão e exclusão; seleção dos textos e sistematização das informações encontradas; e organização e análise dos dados; todos os dados foram coletados, processados, analisados e descritos entre os meses de agosto e novembro do ano de $2020^{10} \mathrm{e}^{11}$.

As palavras chaves utilizadas na busca de dados, foram pré-estabelecidas com
A presente pesquisa

trata-se de uma

revisão sistemática

da literatura, de

característica

exploratória, na

qual buscou gerar

dados quantitativos

e qualitativos das

amostras coletadas.

Esta pesquisa utilizou

de procedimentos

técnicos, que

possibilitasse reunir

informações e dados

que auxiliasse na

fundamentação

teórica, delimitação

da problemática, que

atendesse a hipótese

estabelecida e ao

objetivo proposto. base em outros trabalhos realizados com a mesma temática, sendo: gravidez na adolescência, promoção de saúde, assistência de enfermagem, implicações da gravidez, riscos à saúde maternal, riscos à saúde neonatal e cuidados de enfermagem. Após a delimitação da questão a ser abordada e a definição das palavras chaves, a etapa seguinte consistiu na seleção das bases de dados bibliográficos, capazes de fornecer informações adequadas para o objetivo desta pesquisa. A partir de então, a coleta dos trabalhos correspondentes a temática da pesquisa, procedeu-se no portal de periódicos CAPES, no Scientific Electronic Library Online (SciELO) e no Google acadêmico (Google Scholar), para realizar o levantamento de toda a literatura pertinente ao tema, através dos descritores (palavras chaves) pré definidos.

Os critérios de inclusão (CI) se delimitaram pela seleção de artigos que estivessem sidos publicados no período de dez anos, o que corresponde de 2010 a 2020, para que houvesse uma percepção mais atual referente ao tema. Foram incluídas também as publicações que atendiam aos descritores utilizados e de acesso aberto. Para concluir a seleção, optou-se por selecionar apenas artigos brasileiros escritos na língua portuguesa e publicados em periódicos revisados por pares. Os critérios de exclusão (CE) utilizados foram artigos que não estivessem escritos na língua portuguesa e que não tenham sido revisados por pares, para que se facilitasse a compreensão dos pesquisadores e artigos que fugissem da temática abordada e dos descritores utilizados nas bases de dados. Foram também excluídos estudos que não correspondessem aos anos de busca selecionados e que não estivessem disponíveis para acesso gratuito.

Feita a busca das fontes utilizadas na pesquisa, fez-se então a leitura prévia do resumo deste material, com a finalidade de selecionar aqueles que mais se adequavam a temática da pesquisa. Após a seleção e leitura do material, realizou-se o fichamento dos mesmos. Neste momento, selecionaram-se as partes mais importantes do material escolhido, que atendiam 
ao objeto da pesquisa e se encaixavam no plano de pesquisa.

A organização e análise dos dados procederam-se conforme seguia as etapas propostas para a pesquisa sistemática da literatura, sendo a extração dos dados feitos mediante o levantamento bibliográfico preliminar e posterior elaboração do plano provisório da pesquisa. Para este feito, utilizou o software Excel ${ }^{\odot}$ (2016) para tabulação dos artigos encontrados e realização da análise qualitativa. Além disso, aplicou-se análise quantitativa dos artigos selecionados das bases de dados, mediante as aplicações dos CE e CI.

\section{RESULTADOS}

No levantamento bibliográfico preliminar, realizado nas bases de dados com as palavras chaves descritas anteriormente, foram encontrados 178 artigos, dos quais, após uma filtragem prévia, foram selecionados 128 artigos para compor a amostragem do estudo. Através dos critérios de inclusão e exclusão, foram analisados apenas

\section{Tabela 1. Síntese dos estudos encontrados nas bases de dados consultadas.}

\section{Autores}

12

13

14

15

16

17

18

19

20

21

22

23 Pesquisa de campo com realização de entrevistas

6

24

25

Tipo de estudo

Descritivo e caráter exploratório

Exploratório e abordagem qualitativa

Observacional e transversal

Qualitativo, com abordagem bibliográfica

Exploratório e abordagem qualitativa

Revisão sistemática da literatura

Quantitativo e abordagem exploratória

Revisão integrativa e natureza qualitativa

Exploratório descritivo

Revisão sistemática da literatura

Observacional e transversal

Revisão sistemática da literatura

Observacional e transversal

Observacional e analítico

\section{Síntese do estudo}

Analisou as práticas do enfermeiro na prevenção da gravidez precoce

Identificou e analisou as consequências objetivas e subjetivas de uma gravidez em adolescentes

Investigou a importância da gravidez na adolescência como um problema de saúde

Analisou os fatores determinantes da evasão escolar decorrente da gravidez precoce

Investigou as percepções de puérperas adolescentes sobre a assistência da enfermagem

Sistematização da assistência de enfermagem, com a intenção de uso por profissionais da área

Identificou as ações utilizadas pelos enfermeiros para a prevenção da gravidez na adolescência

Buscou conhecer acerca do papel do enfermeiro na assistência prestada às adolescentes gestantes

Identificou as principais consequências da gravidez enfrentadas pelas adolescentes

Avaliou o impacto da gravidez na primeira fase da adolescência sobre parâmetros clínicos

Avaliou a associação entre gravidez de adolescentes e a ocorrência de nascidos vivos de baixo peso, prematuridade e cesariana

Analisou os fatores de risco para mortalidade fetal e infantil, com atenção especial ao papel da gravidez na adolescência nessa relação

Avaliou as complicações relacionadas à gravidez na adolescência

Identificou os fatores associados à gestação na adolescência

Analisou a associação da gravidez na adolescência com prematuridade
15 artigos, os quais atendiam a proposta deste estudo. Dos quinze artigos analisados, a maior parte corresponde ao tipo de pesquisa observacional de caráter transversal e revisão sistemática da literatura. Em relação ao período de publicação dos estudos selecionados, a maior ocorrência foi para o ano de 2010, com 26,7\% artigos selecionados. $\mathrm{O}$ material selecionado foi organizado e submetido a leitura crítica, sendo feito uma sistematização individual, conforme demonstrado na Tabela 1.

\section{DISCUSSÃO}

O processo de enfermagem consiste em uma série de etapas, como por exemplo: coleta de informações multidimensionais sobre as condições de saúde, identificação de situações que requerem intervenções de enfermagem, planejamento de intervenções necessárias, implementação e avaliação de intervenções, entre outras ações, cujo objetivo é fornecer a comunidade um serviço de modo a comtemplar suas singularidades ${ }^{17}$. Quando trazemos está sistematização para o âmbito da gravidez na adolescência e os cuidados maternal e neonatal, o profissional de saúde, através da assistência de enfermagem, vai além dos cuidados com higiene do recém-nascido e administração das medicações prescritas ${ }^{16}$.

Este processo do trabalho de enfermagem na assistência maternal e neonatal, muitas das vezes não é reconhecido ${ }^{16}$. Corroborando à está discussão, alguns autores abordam o quanto é indispensável este profissional na assistência de enfermagem junto a gravidez na adolescência, promovendo cuidados gerais e locais, avaliando o estado físico e mental da adolescente, observando questôes como o repouso, deambulação, alimentação e preparo da paciente para a alta hospitalar ${ }^{18}$. Soma a isto, também, as orientações quanto aos cuidados com as mamas, com o períneo, atividade sexual, consulta puerperal, planejamento familiar e acompanhamento da saúde do bebề ${ }^{19}$.

É possível pressupor que, a maioria das adolescentes grávidas não possuem 
conhecimentos sobre as complicações e consequências que a gravidez nesta fase da vida pode acarretar ${ }^{20}$. Frente a isso, é perceptível a importância dos profissionais de saúde, em especial os enfermeiros, que por estarem mais próximos dos pacientes, possuem um papel de educação, orientação e encorajamento sobre as adolescentes, sanando quaisquer dúvidas. Isto só reforça o que já vinha sido dito e demonstra quão fundamental é a assistência de enfermagem na gravidez na adolescência.

A gravidez na adolescência, como já foi abordado anteriormente no presente trabalho, é a causa de diversas mudanças físicas, sociais e psicológicas na vida da adolescente. De maneira geral, a gestão é classificada de risco biológico, principalmente para as mães adolescentes ${ }^{15}$. Nesta fase da vida, o corpo feminino ainda se encontra em processo de desenvolvimento, principalmente os órgãos reprodutivos, que após um período de maturidade estão prontos para se reproduzirem normalmente, sem risco de saúde à gestante e o bebê $\hat{~}^{13}$.

Analisando os resultados expostos por alguns trabalhos consultados nas bases de dados, estes revelaram alguns riscos/ complicações clínicas maternal e neonatal devido a gravidez na adolescência. A compilação dos dados permitiu destacar pelo menos seis e sete riscos que podem inferir a mãe adolescente e o recém-nascido, respectivamente. São estes: risco maternal (anemia, hemorragia pós-parto, pré-eclâmpsia, hipertensão induzida pela gestação, diabetes e infecção urinária/ vaginal); risco neonatal (parto prematuro, baixo peso ao nascer, pequeno para a idade gestacional, vitalidade deprimida, malformação neonatal, óbito neonatal e retardo do crescimento intrauterino) ${ }^{6,21}$, $22 \mathrm{e} 23$.

Outros estudos corroboram com a presente discussão, e salientam que os riscos neonatais e maternais, relacionados à gravidez na adolescência, sugerem múltiplas interferências, a constatar pelo núcleo familiar, inserção social, situação conjugal e estado de saúde da paciente ${ }^{24}$
Com relação aos riscos

à saúde maternal e

neonatal, é relatado

que a adolescência não

é um fator agravante

para os maus

resultados obstétricos

obtidos em seu

estudo. $O$ percentual

de abortamento,

prematuridade,

mortalidade maternal

e/ou neonatal, são

características que

envolve uma série de

fatores, não somente a

adolescência. Atrelado

a isso, as condições de

assistência pré-natal,

por parte da equipe

de saúde, é um ponto

que deve ser levado em

consideração. e25. Não obstante disto, têm-se também a possibilidade de desenvolver problemas sociais e familiares, que vale ser citado nesta discussão. Esses fatores, costumam levar a consequências como o abandono escolar e dificuldade de frequentar a escola durante e/ou após a gravidez, pois, as mães adolescentes enfrentarão desafios relacionados às responsabilidades maternas nas atividades escolares ${ }^{15}$.

Com relação aos riscos à saúde maternal e neonatal, é relatado que a adolescência não é um fator agravante para os maus resultados obstétricos obtidos em seu estudo. O percentual de abortamento, prematuridade, mortalidade maternal e/ou neonatal, são características que envolve uma série de fatores, não somente a adolescência. Atrelado a isso, as condições de assistência pré-natal, por parte da equipe de saúde, é um ponto que deve ser levado em consideração ${ }^{14}$.

Antes de falarmos sobre a prevenção dos riscos à saúde da mãe e do recém-nascido, é importante destacar a relevância da educação em saúde como prevenção da gravidez na adolescência, sendo está uma ação primária na prevenção dos riscos que podem vim acometer tanto a mãe como o recém-nascido. A educação sexual possibilita resultados positivos por meio da participação, reflexão e compreensão da importância da vida sexual responsável e da autoproteção entre os pares adolescentes. $\mathrm{O}$ trabalho da orientação e de educação na promoção da saúde dos adolescentes nos centros de Saúde da Família, é a ação mais eficiente na prevenção de uma gravidez precoce, e consequentemente, riscos futuros a saúde da mãe e do recém-nascido ${ }^{12}$.

\section{CONCLUSÃO}

O profissional de saúde, através da assistência de enfermagem, possui um papel fundamental de aconselhamento, educação e tomada de medidas preventivas. Os riscos que podem acometer a mãe adolescente e o recém-nascido, são, na sua grande maioria, da ordem biológica, e possuem uma interação de múltiplos fatores, 
como a situação social da mãe, componente familiar em que ela está inserida e o estado de saúde de ambos. Soma-se a isto, a gravidez na adolescência pode, também, gerar consequências como o desamparo e abando da criança, problemas emocionais relacionados a mãe, afastamento escolar, o desemprego ou a perda de perspectivas futuras no âmbito profissional, e por fim, a multiparidade em um curto período de tempo, o que pode vim agravar toda a situação descrita no presente estudo.

\section{REFERÊNCIAS}

1. Ximenes Neto FRG, Dias MSA, Rocha J, Cunha ICKO. Gravidez na adolescência: motivos e percepções de adolescentes. Rev Bras Enferm. 2007; 60(3):279-285.

2. Dadoorian, D. Gravidez na adolescência: um novo olhar. Psicologia: Ciência e Profissão. 2003; 23(1):84-91.

3. Gonçalves H, Machado EC, Soares ALG, Camargo-Figuera FA, Seering LM, Mesenburg MA, et al. Início da visa sexual entre adolescentes (10 a 14 anos) e comportamentos em saúde. Rev Bras de Epidemiol. 2015; 18(1):1-18.

4. Santos CAC, Nogueira KT. Gravidez na adolescência: falta de informação? Adolescência \& Saúde. 2009; 6(1):48-56.

5. Oyamada LH, Mafra PC, Meireles RA, Guerreiro TMG, Caires Júnior MO, Silva FM. Gravidez na adolescência e o risco para a gestante. Braz J Surg Clin Res. 2014;6(2):38-45.

6. Azevedo WF, Diniz MB, Fonseca ESVB, Azevedo LMR, Evangelista CB. Complications in adolescente pregnancy: systematic review of the literature. Einstein. 2015; 13(4):618-626.

7. Pariz J, Mengarda CF, Frizzo GB. A atenção e o cuidado à gravidez na adolescência nos âmbitos familiar, político e na sociedade: uma revisão da literatura. Saúde e Sociedade. 2012; 21(3):623-636.

8. Freitas $\mathrm{CL}$, Silva JL, Galvão RC, Leonardo MEM, Pereira JB, Vale AJM. Definição do perfil sociodemográfico e reprodutivo de adolescentes grávidas de uma maternidade pública. Adolescência \& Saúde. 2016; 13(2):60-66.

9. Saviani-Zeoti F, Petean EBL. Apego materno-fetal, ansiedade e depressão em gestantes com gravidez normal e de risco: estudo comparativo. Estudos de Psicologia. 2015; 32(4):675683.

10. Mendes KDS, Silveira RCCP, Galvão CM. Revisão integrativa: método de pesquisa para a incorporação de evidências na saúde e na enfermagem. Texto \& Contexto. 2008; 17(4):758764.

11. Galvão MCB, Ricarte ILM. Revisão sistemática da literatura: conceituação, produção e publicação. Logeion: Filosofia da Informação. 2019; 6(1):1-13.

12. Gurgel MGI, Alves MDS, Moura ERF, Pinheiro PNC, Rego RMV. Desenvolvimento de habilidades: estratégia de promoção da saúde e prevenção da gravidez na adolescência. Rev Gaúcha Enferm. 2010; 31(4):640-646.

13. Taborda JA, Silva FC, Ulbricht L, Neves EB. Consequências da gravidez na adolescência para as meninas considerando-se as diferenças socioeconômicas entre elas. Cadernos Saúde Coletiva. $2014 ; 22(1): 16-24$.

14. Guanabens MFG, Gomes AM, Mata ME, Reis ZSN. Gravidez na adolescência: um desafio à promoção da saúde integral do adolescente. Rev Bras Edu Med. 2012; 36(2).

15. Rodrigues LS, Silva MVO, Gomes MAV. Gravidez na adolescência: suas implicações na adolescência, na família e na escola. Revista Educação e Emancipação. 2019; 12(2):228-252.

16. Nóbrega LLR, Bezerra FPF. Percepções de puérperas adolescentes frente à assistência de enfermagem no alojamento conjunto. Revista Rene (Online). 2010; 11(1):42-52.

17. Carvalho EC, Bachion MM. Processo de enfermagem e sistematização da assistência de enfermagem: intenção de uso por profissionais de enfermagem. Rev Eletrônica Enferm. 2010; 11(3):466.

18. Ribeiro VCS, Nogueira DL, Assunção RS, Silva FMR, Quadros KAN. Papel do enfermeiro da estratégia de saúde da família na prevenção da gravidez na adolescência. Revista de Enfermagem do Centro Oeste Mineiro. 2016; 1(6):1957-1975.

19. Moreira TMA, Sousa DF, Silva SET, Santana WJ, Luz DCRP. 0 papel do enfermeiro na assistência prestada às adolescentes grávidas. Revista e-ciência. 2016; 4(1):43-53.

20. Araújo RLD, Rodrigues ESRC, Oliveira GG, Sousa KMO. Gravidez na adolescência: consequências centralizadas para a mulher. Temas em Saúde. 2016; 16(2):567-587.

21. Bouzas ICS, Cader SA, Leão L. Gravidez na adolescência: uma revisão sistemática do impacto da idade materna nas complicações clínicas, obstétricas e neonatais na primeira fase da adolescência. Adolescência \& Saúde. 2014; 11(3):7-21.

22. Santos NLAC, Costa MCO, Amaral MTR, Vieira GO, Bacelar EB, Almeida AHV. Gravidez na adolescência: análise de fatores de risco para baixo peso, prematuridade e cesariana. Ciência \& Saúde Coletiva. 2014; 19(3):719-726.

23. Oliveira EFV, Gama SGN, Silva MFP. Gravidez na adolescência e outros fatores de risco para mortalidade fetal e infantil no município do Rio de Janeiro, Brasil. Cadernos de Saúde Pública. 2010; 26(3):567-578.

24. Moura B, Saldanha M, Lopes M, Guaraná M, Mendes N, Simões R, et al. Gravidez na adolescência: fatores associados e resultados perinatais em uma maternidade-escola do Rio de Janeiro. Adolescência \& Saúde. 2011; 8(1):15-20.

25. Martins MG, Santos GHN, Sousa MS, Costa JEFB, Simões VMF. Associação de gravidez na adolescência e prematuridade. Revista Brasileira de Ginecologia e Obstetrícia. 2011; 33(11):354-360.

26. Azevedo AEBI. Guia prático de atualização: prevenção da gravidez na adolescência. Sociedade Brasileira de Pediatria. Departamento cientifico de adolescência. Janeiro de 2019. 